\title{
Scaffolding Visually Cluttered Web Pages to Facilitate Accessibility
}

\author{
Alison Lee \\ IBM TJ Watson Research Center \\ 19 Skyline Drive \\ Hawthorne, NY 10532
}

\begin{abstract}
Increasingly, rich and dynamic content and abundant links are making Web pages visually cluttered and widening the accessibility divide for the disabled and people with impairments. The adaptations approach of transforming Web pages has enabled users with diverse abilities to access a Web page. However, the challenge remains for these users to work with a Web page, particularly among people with minimal Web experience and cognitive limitations. We propose that scaffolding can allow users to learn certain skills that help them function online with greater autonomy. In the case of visually cluttered Web pages, several accessibility scaffoldings were created to enable users to learn where core content begins, how text flows in a part of a Web page, and what the overall structure of a Web page is. These scaffoldings expose the elements, pathways, and organization of a Web page that enable users to interpret and grasp the structure of a Web page. We present the concept of an accessibility scaffolding, the designs of the scaffoldings for visually cluttered pages, and user feedback from people who work with our target end-users.
\end{abstract}

\section{Categories and Subject Descriptors}

H.5 [Information Interfaces and Presentation]: User Interfaces - interaction styles, user-centered design, voice I/O. Hypertext/ Hypermedia - navigation and user issues. I.7 [Document and Text Processing]: Document Preparation - multi/mixed media. Document Capture - document analysis.

\section{General Terms}

Design, Experimentation, Human Factors.

\section{Keywords}

Accessibility, design space, dynamic diversity, interaction design, page segmentation, scaffolding, GUI button interface, recorder interface, speak text, and Web.

\section{INTRODUCTION}

Increasingly, rich and dynamic content and abundant links are making Web pages visually cluttered. As a result, they are widening the accessibility divide and posing significant problems for those who have multiple and varying forms of visual, cognitive, and motor impairments. Efforts to reduce clutter by removing content (e.g., ad blocking) or by extracting content can improve Web accessibility but they introduce other kinds of problems [5]. Spe- cifically, such approaches are not robust across a broad set of Web pages and services [7]. Second, content removal takes away from a Web page's inherent look and feel. When performed automatically or by an intermediary, the act of excerpting a page's content raises concerns over fair-use of the publisher's copyrighted material. Finally, publishers argue that accessing their content while blocking advertisements is tantamount to freeloading because such consumers are getting content without compensating the publishers who use advertisements to help make the content available. Hence, content removal and content extraction approaches pose a myriad of technical, legal and social challenges.

A promising alternative to content removal and content extraction allows users with diverse abilities to apply one or more transformations such as text enlargement or keyboard adjustment (i.e., adaptations) $[7,9,10]$. While these users can see and interact with a Web page, their lack of Web sophistication or difficulties with keeping focus or attention impacts their ability to work with a Web page. We propose to build on the adaptations approach by adding scaffolding capabilities that allow users to learn certain skills for functioning online with greater autonomy. In the case of visually cluttered Web pages, the accessibility scaffoldings enable users to learn where core content begins, interpret the text flows in a part of a Web page, and grasp the overall structure of a Web page is.

In the following section, we review techniques and approaches to Web accessibility with the goal of identifying the key innovations of our approach. Then, we present the scaffolding approach to accessibility and its instantiation for scaffolding visually cluttered Web pages to facilitate accessibility. We conclude with informal feedback from individuals who work with our end-users to access the Web (e.g., older adults, individuals with literacy problems).

\section{RELATED WORK}

The content manipulation approach for addressing the visual clutter problem exists in various forms including popup removal resources, content extraction tools, and content adaptations. They each have problems that limit their broad appeal, usability, and effectiveness. For example, while the adaptation approach has enabled users with diverse needs to use the Web by providing visual transformations and keyboard and browser adaptations, they do not effectively service users with cognitive limitations (e.g., content comprehension, attention deficit) or users with confidence issues associated with low computer literacy [7, 14]. Adaptations do not address issues such as learning, the development of an appropriate conceptual model, and the need for personal support. Such issues have been of concern to those in the educational domain and for which scaffolding is a cornerstone technique that has been developed and used [2].

Assistive technologies enable people with special needs to use computers [1, 8]. However, they do not improve the individual's ability to perform and complete tasks and lack features that allow their users to deal easily with visually cluttered pages. More importantly, they focus on individuals with particular disabilities rather than individuals such as older adults with dynamic abilities that impact their interactions with computers [4, 14]. 
The approaches mentioned thus far, including accessibility scaffolding, focuses on end-users. There are, however, initiatives that help designers create accessible and usable Web sites through guidelines, authoring tools, and evaluation tools [3, 7, 12]. There are also initiatives that call on an early focus during design $[4,11]$ for dynamic diversity. One such proposal seeks a radically different paradigm and methodology to design that is as near to the universal accessibility ideal as is possible. While well-intentioned and viable, we see their impact and contributions to be more long-term and unable to overcome current limitations, problems, and legacy of Web pages and sites.

In the educational and learning research and practice, the concept of scaffolding is a well-known and important [13]. Scaffolding technologies, such as tutoring systems, microworlds, and computer-supported learning environments, aid learning by providing a structure that keeps learners productive without destroying initiative, motivation and resourcefulness [2]. They assist and encourage learners to carry out the task and to gradually shift responsibility for performing the task to the learner. Accessibility scaffoldings function similarly but are different in two ways. First, the scaffolds themselves focus on supporting or developing a skill associated with particular accessibility limitations related to various disabilities or changing abilities. Second, the scaffoldings use adaptations to enable users to get on the Web. Adaptations (e.g., [9]) by themselves neither provide the structures for supporting learning nor prevent users from straying too far off-the-path.

\section{ACCESSIBILITY SCAFFOLDING}

Scaffolding to facilitate accessibility is appealing because it is used in practice by learning centers that support such user groups. Through coaching, teaching, and strategy sharing, users learn techniques that guide and assist them with ways to deal with such pages. However, once outside of these support environments, users lack the scaffolds that support and reinforce the ways to deal with such pages. Extending the adaptations approach with scaffolding helps address the accessibility skill and literacy needs associated with strategies for dealing with, for example, visually cluttered pages. The scaffolds provide clear directions, help keep learners from straying too far off a pathway, and point learners to worthy source while adaptations provide the accessibility enablers to address the diverse visual and physical abilities of the users.

Accessibility scaffoldings, unlike assistive technologies that make it possible for people with special needs and disabilities to function, empower individuals and enhance the user experience. Some of these scaffolds can disappear after it has served its purpose but they can also remain in place to assist in perpetuity. However, they should not be viewed as prosthetics that replace the individual's ability to perform but should be viewed as a structure that develops certain skills and aids in self-independent performance of tasks requiring those skills. Also, scaffolding is more than just facilitation of task performance, it explicitly highlights and develops specific skills (i.e., finding core part of a Web page).

The design of the present accessibility scaffoldings exploited patterns of use of the Web adaptation software [9]. This software includes 12 sets of adaptations combining visual adaptations such as image enhancements, redundant presentations such as speak text, keyboard adjustment, and enlargement of browser controls. It was distributed to the members of a national organization working with seniors in October 2002 (known as User Group 1). A study [6] of usage about five months after its availability revealed that all adaptations were used by some people. The single-most used adaptation ( $40 \%$ of the population) was 'speak text', wherein the user points the mouse at that portion of the page they want to have read aloud. Other adaptations, used by one third of this population, are font enlargement, font style changes, banner text and changing color contrasts. The software was also made available to adults with basic literacy and individuals with developmental disabilities (known as User Group 2). While not designed for such groups, this software enabled many of these users to venture independently onto the Web [9]. In particular, users with literacy problems and difficulties in reading well used the 'speak text' feature to follow along as the computer reads the text on the screen [9].

\section{SCAFFOLDING CLUTTERED PAGES}

Pre-design interviews with advocates of users with diverse abilities revealed that it is neither intuitive nor easy for the users to analyze and assimilate the overview for a page. They suggested that scaffoldings that make the elements, text flows, and organization of the Web page more evident could be valuable in facilitating accessibility to cluttered Web pages. Herein, we present three scaffoldings, each representing a point in their respective design subspace that focus on certain accessibility skills to be acquired in dealing with cluttered Web pages. In particular, we focus on where core content begins, how text flows in a Web page, and what the overall structure of a Web page is using information about a Web page's organization and its components (i.e., page-segments).

\subsection{Position, Speak, Highlight First Core Text}

Early discussions with people serving individuals in User Group 2 described the difficulties their users have with using the Web. Their users are distracted by the clutter of text and images and have difficulties with identifying the area of the Web page to attend to. Similarly, the people working with seniors indicated that banner advertisements, in particular, tend to captivate their users to the exclusion of the content present in the rest of the page. In both cases, explicit guidance to look in a certain spot or to scroll down to look for particular content must be given.

These difficulties suggested that one use of the page-segment scaffold is to automatically reposition, highlight, and speak out the first piece of content in the core region. In jumping to the start of the core region, we proactively navigate the user's attention to the core region and bypass the banner elements like the advertisements and menus that distract and confuse them. Then, in highlighting the text, we explicitly orient the user and call attention to a particular element on the page. By speaking the highlighted text, we actively reinforce the explicit guidance of the user's attention to the beginning of the main content of the page.

\subsection{Speak Content Using Player Interface}

Seniors made extensive use of the 'speak text' adaptation to supplement what they were viewing which greatly improved their ability to access the content despite their various visual impairments. For the people with basic literacy, speak text helps them work through their reading and literacy difficulties while hearing the text read aloud. Thus, in the design of the second scaffolding to help the user follow the text flow on a Web page, we provided a player interface that permits users to select the text to be spoken using the 'speak text' adaptation (see Figure 1). This player interface appears next to the Web page. Clicking the play button (i.e., light-grey square), skip-to-previous/next-text-in-a-page-segment button (i.e., single left/right arrow), or fast-forward/backward-tonext-page-segment button (i.e., double down/up arrow) caused the appropriate text to be selected and read out. The selected text and 'speak-text' route within and between page segments are guided by the page-segment scaffold. As the text is read out, its location on the Web page is highlighted to enable the user to see what is being read and to follow along. The text read-out is preceded, in some cases, with a narrative component that provides orientation (e.g., 'This is the first piece of text.'). To separate this narrative text from the Web page text, it is spoken in a different gender voice. Functionality from the first scaffolding is also available to users of this 


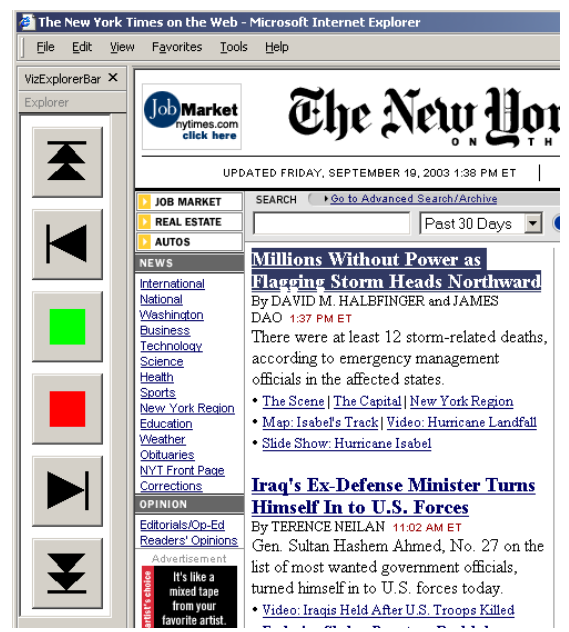

Figure 1: Player interface to speak the text, in order, for the New York Times page guided by the page-segment scaffold.

interface (i.e., on loading a new Web page, the first piece of text in a beginning core page segment is highlighted and read out).

\subsection{Page-Segments Exposed through Buttons}

The clutter of images, text, and other visual elements present in a Web page do make it difficult for users. For example, some seniors with little knowledge of the Web or users with poor visual abilities have difficulties seeing the forest from the trees and identifying the macro page structure. With proper scaffolding, these users are able to bring problem-solving skills to bear in dealing with a visually cluttered Web page. Thus, the third scaffolding attempts to supplement the user's view of a Web page with a simpler and less attention-grabbing view that would enable them to grasp the larger structure of a Web page. The page-segment scaffold contains proportional-sized buttons representing the key segments in a Web page (see Figure 2). When users navigate to new Web pages, the corresponding visual page segment structure appears alongside the Web page in the Web browser. As with the first scaffolding, the text content associated with a beginning core page segment is brought into view, highlighted and spoken out.

Clicking a button highlights the start of the page-segment represented by the button and, if necessary, brings the portion of the Web page into view in the Web browser. The buttons are kept visually simple to make them viewable and readable using a large font size and the first few words of the first text of the designated segment. Hovering over the button with the mouse results in an

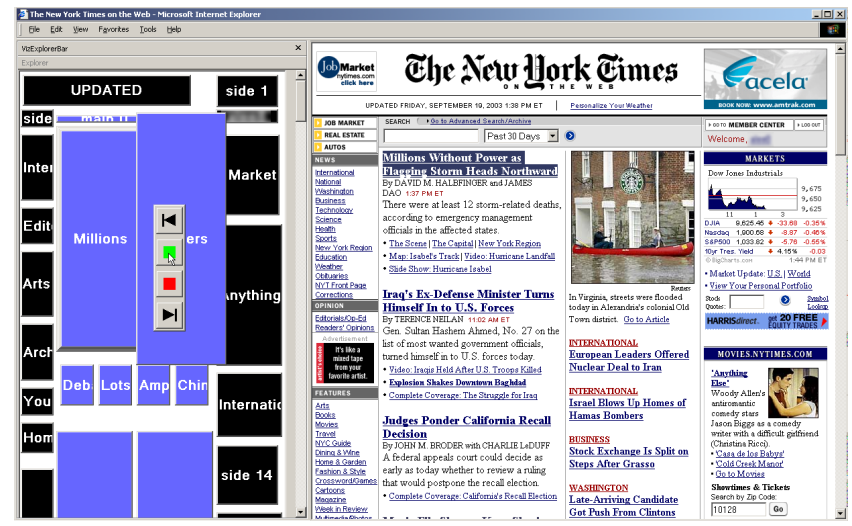

Figure 2: Button interface which renders page segments as buttons for the New York Times page. enlargement of the button for better viewing of the words. The darker buttons are for page segments located at the periphery of Web page. Lighter colored buttons designate page segments whose content is located in the core region of the Web page by the page segmentation heuristic. As with the second scaffolding, users can invoke the player interface. Specifically, right-clicking on the button brings up the player interface as a popup menu. Selection of the menu item results in the current text or next/previous text in the button's page segment to be spoken out.

This interface provides greater user flexibility in choosing which page segment's textual content is read out. It supports increased user sophistication and user skill to navigate the Web page's content. More importantly, this interface reveals the Web page's structure and provides capabilities for exploring the textual content of the Web page's segments. These advanced concepts builds on concepts introduced by the first two scaffoldings.

\subsection{Implementation}

The implementation of the three scaffoldings made extensive use of a novel page-segmentation heuristic. This heuristic reconstructs the Web page layout and identifies the main regions and segments of a page using whitespace and regions as the primary criteria (see Figure 3). It takes as input, the Document Object Model (DOM) tree information along with information about the graphical layout (i.e., position and size) of the DOM elements. The heuristic uses four passes to reconstruct the Web page's layout. First, it finds the visual blocks of the Web page. Second, the visual blocks are separated into those belonging to the core region and border regions. Third, it determines whitespace separators between visual blocks in each of the core and peripheral regions. Finally, visual blocks are aggregated into larger units based on depth in containment hierarchy, separator size and visual properties of the visual blocks.

\section{FEEDBACK AND RECOMMENDATIONS}

Full functioning versions of the three designs were shown to people working with seniors (User Group 1) and people working with users with diverse development disabilities (User Group 2). They consisted of three instructors and one telephone support person from the first group and five people from the second group (i.e., the coordinator of instructional program, three instructors, and IT support person). We sought feedback from these people first because of their experience in working with end-users with diverse abilities and in introducing the Web adaptation software to these end-users.

Overall, our informants were uniformly positive about the first two scaffoldings (i.e., core content and speak text linearly in Web page with player interface). The seniors' informants indicated that aside from the utility of taking the users explicitly to the start of the core content, this scaffolding bypasses difficult and problematic content that generally captivated and confused the seniors. The second

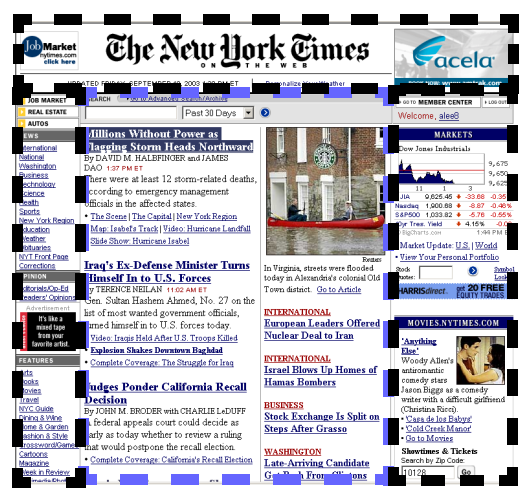

Figure 3: A Web page's content is laid out in blocks with "core" content surrounded optional by "peripheral" content. 
group of informants recommended that the spoken text be shorter; on the order of a sentence construct. While the spoken message length was not commented upon by the people in the first group, prior studies indicate that shorter spoken text is desirable [14].

The informants for User Group 2 particularly favored the player scaffolding. First, the interface was simpler and was familiar, for some of their users. Second, the simpler interaction of a button press compared to pointing to text in the 'speak text' adaptation enabled the user to hear and see the spoken text. Third, the structured and linear walk-through of the textual content on the page was considered most beneficial and not constraining. In fact, they suggested the elimination of the two fast forward and backward buttons (i.e., double arrows) because they felt that the functionality and its differentiation with the other two skip buttons were lost on their users, particularly the developmentally disabled. Even for the adults in their basic literacy program, this ability to systematically move to and speak out the text in the document was valuable for learning to read and for learning english. In terms of the narrative component preceding the 'spoken text', they felt the concept was potentially useful but not for indicating whether the spoken text was the last item. Our informants for the seniors also viewed the simple interface of the second scaffolding to be useful and, particularly, for those users with visual and motor impairments. They did indicate that the iconic buttons and player metaphor could be problematic for those seniors unfamiliar with the icons and metaphor.

Both groups of informants raised concerns about the potential difficulties for their constituencies to make the connection between the visual page-segment representation and the macro structure of the page in the third scaffolding. The informants for the developmentally disabled also felt there were insufficient words for the label. In some cases, the selection of the first words in the first sentence of a segment was not very meaningful. The informants for the seniors indicated that the interface may be too complex for some to see, to comprehend, or to interact with despite the reduction to simple boxes and words. However, both groups did agree that the general notion of a supporting structure was valuable and provided several suggestions. One of the seniors' informants, who was herself a senior, was intrigued by this design but indicated that she was more sophisticated as a computer and Web user.

The feedback indicates that using the page-segment scaffold in the first two scaffolding to facilitate and assist the end-users was valuable. However, rendering it literally and in a visual form in the buttons-map scaffoldings is problematic. We are exploring other more advanced scaffolding in which the page-segment serves as a behind-the-scenes guide.

\section{CONCLUSION}

The Web and its many visually cluttered Web pages presents a daunting challenge for users with diverse abilities. In addition to their varying and dynamic impairments, such users lack the sophistication, abilities, and support needed to access and interact with the Web. We argue that facilitating accessibility to Web pages include not only providing technologies and software such as adaptations, but also scaffoldings that provide structure, supports and routes to gradually and independently access Web content. We demonstrate how the basic concept of scaffolding can be applied to an instance of an accessibility problem (i.e., visually cluttered Web pages) in three example scaffolding designs. Feedback on the designs yielded positive comments and many suggestions. Clearly, the idea of providing structure and guidance resonated with our user supporters. Their suggestions and directions addressed particular issues and disabilities experienced by their constituencies.

Our exploration points to several directions for future work. First, the design space for scaffoldings is rich but unmapped. Second, questions about what the appropriate scaffold structure is and what the requirements and prioritization of the flexibility and functionality provided in a scaffolding need to be further explored. Finally, studies and observations of use with end-users are needed to shed light on the variety and form of the scaffoldings for accessibility.

\section{ACKNOWLEDGMENTS}

I would like to acknowledge the contributions of the Web Adaptation Technology team; particularly Cal Swart and Beth Tibbitts. As well, Dan Oppenheim assisted with Microsoft's explorer bar, BHO and connector mechanisms. Finally, Rob Farrell, Doug Gordin and Kevin Singley gave valuable insights on educational scaffolding.

\section{REFERENCES}

[1] C. Asakawa and T. Itoh. User Interface of a Home Page Reader. In Proceeding of the Third International ACM Conference on Assistive Technologies (ASSETS'98), ACM Press:New York, pp. 149 - 156, 1998.

[2] J. Bransford, A.L. Brown, and R.R. Cocking (eds). Technology to Support Learning. How People Learn: Brain, Mind, Experience and School, pp. 194-218, National Academy Press, 2000.

[3] Center for Information Technology Accommodation. Section 508. The Road to Accessibility, 2002. Available at http:// www.section508.gov/.

[4] P. Gregor, A. F. Newell, M. Zajicek. Designing for Dynamic Diversity - Interfaces for Older People. In Proceeding of ASSETS 2002, New York: ACM Press, pp. 151-156, 2002.

[5] S. Gupta, G. Kaiser, D. Neistadt and P. Grimm. DOM-based Content Extraction of HTML Documents. In Proceeding of the Twelfth International World Wide Web Conference, 2003.

[6] V.L Hanson Facing the Future: Including Elderly Users When Considering Universal Access. In Proceeding of the 2nd International Conference on Universal Access in HumanComputer Interaction, pp. 394 - 398, 2003.

[7] M. Ivory, J. Mankoff and A. Le. Using Automated Tools to Improve Web Site Usage by Users with Diverse Abilities. In Information and Technology: A Web Journal Studying How Technology Affects Society, 3(1), pp. 195-236, 2003.

[8] E.D. Mynatt and G. Weber. Nonvisual Presentation of Graphical User Interfaces: Contrasting Two Approaches. In Proceeding of CHI'94 Human Factors in Computing Systems, New York: ACM Press, pp. 166-172, 1994.

[9] J. Richards, V. Hanson and S. Trewin. Adapting the Web for Older Users. In Proceeding of 2nd International Conference on Universal Access in Human-Computer Interaction, pp. 892 $-896,2003$.

[10] S. Trewin. Extending keyboard adaptability: An investigation. Universal Access in the Information Society, 2(1), Springer Verlag:Heidelberg, pp. 44 - 55, 2002.

[11] G. Vanderheiden. Fundamental Principles and Priority Setting for Universal Usability. In Proceeding of ACM Conference on Universal Accessibility (CUU'00), New York: ACM Press, pp. 32-38, 2000.

[12] Watchfire. Bobby Worldwide, 2002. Available at http:// bobby.watchfire.com/bobby/html/en/index.jsp.

[13] D. Wood, J. C. Bruner, and G. Ross. The Role of Tutoring in Problem Solving. Journal of Child Psychology and Psychiatry, 17 (2), pp. 89-100, 1976.

[14] M. Zajicek. Interface Support for Elderly People with Impaired Sight and Memory. In Proceeding of the 6th European Research Consortium for Informatics and Mathematics Workshop on User Interfaces for All, pp. 177-182, 2000. 